\title{
A Study on Forming a Consensus Measure Space of Macro-seismic Anomalies
}

\author{
Weidan Wang ${ }^{1,2}$, Chongfu Huang ${ }^{1,2, *}$ \\ ${ }^{1}$ State Key Laboratory of Earth Surface Processes and Resource Ecology (Beijing Normal \\ University), Beijing 100875, China \\ ${ }^{2}$ Academy of Disaster Reduction and Emergency Management, Ministry of Civil Affairs \& Ministry of \\ Education, the Peoples' Republic of China, Beijing 100875, China

\section{建立共识性地震宏观异常测度空间的研究} \\ 王蔚丹 ${ }^{1,2}$, 黄崇福 ${ }^{1,2, *}$ \\ ${ }^{1}$ 地表过程与资源生态国家重点实验室(北京师范大学), 北京 100875, 中国 \\ 2 民政部/教育部减灾与应急管理研究院, 北京 100875 , 中国
}

\begin{abstract}
Besides a simple list, the current studies of macro-seismic anomalies are mostly statistical analysis based on single kind of anomaly or several seismic data. Lack of the support of measure space to synthesize macro-seismic anomalies, it is difficult to carry out systematic scientific research. This paper presents the concept of macro-seismic anomalies group, and develop a platform of Internet of Intelligences to push a variety of simulate scenarios of macro-seismic anomalies, which will be judged by a number of experts on the Internet. So that, a consensus of measure space can be established to synthesis quantify macro-seismic anomalies.
\end{abstract}

Keywords: Internet of Intelligences; macro-seismic anomalies group; measure space

\section{摘要}

目前的地震宏观异常研究, 除了简单的罗列, 就是单项异常与震例的简单统计分析, 缺少宏 观综合异常测度空间的支撑, 难以开展系统性

*通讯作者: 黄崇福 hchongfu@bnu.edu.cn
的科学研究。本文提出宏观异常群的概念, 并 研制智联网平台推送各种虚拟的宏观异常情 景, 拟由多位专家分别在网上对宏观异常情景 进行评判, 从而形成共识性的地震宏观异常测 度空间, 实现地震宏观异常的综合量化。

关键词: 智联网; 宏观异常群; 测度空间

1. 引言

大规模的宏观异常, 往往具有强震短临前兆的 性质, 对地震预报有重要的实用意义[1]。在我 国地震预测实践中, 1975年辽宁海城地震、

1976 年云南龙陵地震、1976 年四川松潘地震 等的临震预报成功, 宏观异常起到了十分重要 乃至决定性作用[2]。在现今地震物理预报还没 有过关的情况下, 切实地抓好宏观异常是具有 防震减灾实效的重要途径。

现实是, 近些年来宏观异常甚至没有发挥 以往的作用, 相关研究进展缓慢。一方面原因 是宏观异常数据主要是通过震后调查获得, 震 前获得的数据较少。另一方面, 缺少宏观综合 异常测度空间的支撑, 难以开展系统性的科学 研究。与此同时, 宏观异常的认识和运用主要 由地震专家来完成, 无论是宏观异常的现场核 查, 还是对宏观异常进行宏观把握, 辅助地震 
预报，都离不开专家的参与。

那么如何充分挖掘专家的智慧, 建立宏观 异常测度空间, 为宏观异常的系统化、理论化 研究提供基础, 使其为防震减灾服务, 互联网 的普及, 为我们创造了条件; 智联网的提出 [3] 及其理论的发展、相关应用平台的研发[4-13], 为我们这一目标的实现提供了理论和技术基 础。

为了更好地研究宏观异常与地震的关系, 本文提出宏观异常群的概念, 采用相应指标来 综合度量宏观异常。同时建立相应的智联网平 台, 由专家智能体根据自身的经验知识对虚拟 宏观异常群进行评判并进行模糊输入, 从而建 立地震宏观异常的测度空间。

\section{2. 宏观异常的研究现状和存在的问题}

我国学者对地震前的宏观异常进行了一系列 的相关研究。在《地震宏观异常摘编》一书中 分别对 1937 年菏泽 7.0 级地震、1966 年邢台 地震和 1975 年海城地震等 9 个 6.5 级以上地 震的序列、烈度以及出现的宏观异常现象等进 行了详细的阐述和研究 [14]。祝晔、李荣安对 海城地震宏观前兆时空演化特征与异常机制 进行了研究[15], 刘五洲、车用太对张北-尚义 地震进行宏观异常的震后调查及分析[16], 高 小其等对 2008 年于田 7.4 级地震进行了宏观 异常的调查与分析[17], 许敦煌在 2008 年汶川 地震后对震区进行现场调查[18], 对张小涛等 将从各个渠道获得的 2008 年汶川 8.0 级地震 前后宏观异常现象进行总结分析[19]。张永久 等对汶川地震与松潘一平武地震的宏观异常 进行对比分析发现二者有异有同[20], 付虹等 对云南地区地震的宏观异常特征进行研究 [21], 卢双苓等基于 《中国震例》对宏观异常 与地震的关系进行了统计分析[22]。国外也有 一些人对宏观异常进行了研究, 如 1911 年外 国学者戈利齐恩就注意到震前矿泉动态的变 化, 日本学者曾对鲶鱼进行长期的观测与研究 等[23]。

上述研究或主要积累宏观异常的震例数 据, 在此基础上对异常现象和地震的关系进行 简要分析, 或基于数个震例, 对宏观异常和地 震的关系进行统计分析。但是由于积累的异常 资料有限等原因, 难以得到具有普适性的规
律, 可以肯定的一点是, 大震前一般是有宏观 异常的, 一般具有短临前兆性质, 但是利用其 进行地震预报又是万分困难的。同时大规模的 宏观异常, 既有地震前兆属性, 也有构造活动 异常属性[24]。

由于宏观异常与地震的关系以及其本身 的不确定性、主观性等原因, 只有大规模的群 体性的宏观异常才对地震有指示意义, 针对目 前宏观异常研究都是比较零散的针对个别震 例的现状, 本文提出宏观异常群的概念, 对和 地震相关的宏观异常进行综合度量。在现今的 科技水平下, 宏观异常大都是经验研究, 离不 开专家的参与, 基于已有的宏观异常研究模拟 宏观异常群的场景, 专家通过智联网平台参与 评判, 从而建立共识性的测度空间。以便进行 系统性的探索研究, 为真实的宏观异常的量化 分析和进一步研究宏观异常与地震的关系做 准备。

\section{3. 地震宏观异常的综合度量}

\section{1 地震宏观异常}

宏观异常指人类用感官可直接觉察到的自然 界中的不正常现象, 其中与地震活动密切相关 的现象称其为地震宏观异常 [1]。宏观异常种类 繁多, 表现多样。为了尽可能地囊括所有的异 常类别, 并和微观异常相对照, 一般把宏观异 常系统地分为生物异常、地下流体异常、地球 物理场异常、地质现象异常、气象异常五大类, 如表 1 所示。地下流体宏观异常主要是地下水 异常, 生物异常主要指动物异常。二者是最主 要的两类异常。其中地下水异常主要表现为水 位缓慢上升、下降; 冒泡、水变浑; 水位忽升 忽降; 水位快速上升、下降; 井水突然自流; 地面翻砂冒水; 变色等。动物主要表现为迟针, 呆滞不动, 不怕死, 或惨叫嚎叫, 宁死也要外 逃, 惶惶不安, 成群出现, 慌乱逃跑, 惊叫惊 飞, 不进窝或上下翻腾等。

以往对于比较显著的群发性宏观异常, 一 般用种类多、数量多、范围广、规模大、随时 间剧烈变化等来描述, 即不同宏观异常现象存 在地域上的相关性和时间上的同步性特征。

\section{2 宏观异常群}

宏观异常群是指在一定时空范围发生的 


\section{Risk Analysis and Crisis Response in Big Data Era (RAC-16)}

宏观异常现象构成的情景。选用宏观异常群的 一些参量来综合度量宏观异常。一般来说，指 标的选取要尽可能地科学、详尽、完备、合理, 指标选取质量直接关系到整个结果的可靠性 和正确性。要遵循科学性原则、全面性原则、 可行性原则。

\section{3 宏观异常群的参量表达和参量量化}

根据历史上已有的宏观异常资料, 将宏观 异常用种类数量、分布情况和个体强度这 3 类 参量来进行表达, 具体地, 种类数量指种类数 量和总量, 即出现了哪几类, 共多少起异常; 分布情况主要用空间密集度和时间上的频度 来表示; 个体强度主要是指一起宏观异常表现 的强烈程度。其中, 空间密集度指宏观异常总 量在空间范围的分布密度, 时间频度则表征宏 观异常在时间上的同步性。不同异常现象在空 间上的相关性和时间上的同步性是在宏观异 常研究中尤其要关注的内容, 在本文中, 问了 简化研究, 遵循从简单到复杂的原则, 采用种 类 $V$ 、总量 $T$ 、空间密集度 $D$ 、异常幅度 $I$ 来 建立相应的测度空间。时空演化的整体研究可 以在后续研究中来实现。

在尽可能简化原则的基础上, 种类采用表 1 中 5 大类宏观异常来描述（即生物异常、流
体异常、地球物理场异常、地质现象异常和气 象异常), 取值为 1-5。在一定的时空范围内, 根据已有资料及相关研究, 地震前宏观异常总 量少则 0 , 多则上千。根据以往的震后调查研 究, 其范围在 3-2500 左右。空间密集度即单 位面积内的宏观异常总量。异常幅度, 此指标 主观性较强, 可将宏观异常大致分为一般异常 和重要明显异常。

\section{4. 智联网简介}

每个人, 作为智能体, 或多或少会对某些事物 或环境有比较深刻的认识, 从而可以为解决某 个问题贡献一部分力量。例如, 一个导游可用 相关知识和经验提供关于旅游的一些信息。专 家可帮助别人解决与专业相关的某些问题。地 震预测是一个世界科学难题, 地震相关研究还 处在一个探索阶段, 地震工作者们, 尤其是战 斗在一线的地震专家们, 他们的经验智慧在现 阶段是不可轻忽的。把地震专家们看作高级智 能体, 通过智联网平台把他们的经验知识、判 断等贡献出来, 并加以综合分析处理, 或可为 地震事业添砖加瓦，服务于大众。

设 $A$ 是一个智能体集合, $N$ 是 $A$ 使用的一 个网络, $M$ 是处理 $A$ 所提供信息的模型, 三元 体 $<A, N, M>$ 称为一个智联网, 记为 $\Phi$ 。

表 1. 地震宏观异常分类

\begin{tabular}{|c|c|c|}
\hline \multirow{3}{*}{$\begin{array}{l}\text { 宏观异常 } \\
\text { 大类 }\end{array}$} & 宏观 & 宏观异常种类 \\
\hline & 异常 & \\
\hline & 类别 & \\
\hline \multirow[t]{3}{*}{ 生物异常 } & $\begin{array}{l}\text { 动物 } \\
\text { 异常 }\end{array}$ & $\begin{array}{l}\text { 小动物、家畜、家禽、大牲畜、穴居动物、水生动物、鸟类、鸽子、大动物 } \\
\text { (牛、马、骡、驴、羊、狗、猪、兔、鸡、鸭、鹅、鸽、老鼠、蛇、青蛙、 } \\
\text { 鱼、蚂蚁、蜜蜂、燕子、婴武等) }\end{array}$ \\
\hline & 植物 & 树木、花草、蔬菜 \\
\hline & 异常 & \\
\hline \multirow{3}{*}{$\begin{array}{l}\text { 地下流体 } \\
\text { 异常 }\end{array}$} & 地下 & 井水位上升、井水位下降、泉水流量剧增、泉水流量减少或断流、井 (泉) 水翻花 \\
\hline & 水异 & 冒泡、井 (泉) 水变浑、井 (泉) 水变味、井 (泉) 水有异味、井水面飘油、井水面打 \\
\hline & 常 & 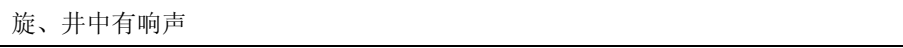 \\
\hline \multirow{2}{*}{$\begin{array}{l}\text { 地球物理 } \\
\text { 场异常 }\end{array}$} & 地声 & -- \\
\hline & 地光 & \\
\hline \multirow{3}{*}{$\begin{array}{l}\text { 地质现象 } \\
\text { 异常 }\end{array}$} & 地鼓 & - \\
\hline & 地裂 & \\
\hline & 塌陷 & \\
\hline 气象异常 & -- & 气温、地温、湿度、气压、降雨 \\
\hline
\end{tabular}


具有观察、演绎、推理和解决问题能力的 个体, 称为智能体。每个具有正常智力水平的 人都可以成为智能体。而地震专家, 因其所具 有的对地震特有的认识、了解, 能做出普通人 无法完成的宏观把握、认知判断等, 成为高级 智能体。感性认识是理性认识的基础, 理性认 识依赖于感性认识。在现今我们还无法正确认 识地震本质和内在规律的情况下, 不断地丰 富、完善已有材料，使之系统化，同时使材料 尽可能地真实, 才有可能随着科技进步和人类 发展在将来攻克地震这个难题。通过一定的模 型对从智能体处得到信息进行处理, 才能进行 智慧的升华。在宏观异常情景评判智联网中, 组成智联网的三元体 $\langle A, N, M>$, 网络 $N$ 是智 联网形成的基础, 智能体 $A$ 主要是地震专家 们, 尤其是对宏观异常有充分接触和深刻认识 的专家们。

\section{5. 宏观异常情景评判智联网}

基于已有的“宏观异常群”概念及其参量表达、 量化, 进行虚拟宏观异常场景的创设, 从而建 立宏观异常情景评判智联网, 通过专家参与情 景评判达到建立共识性地震宏观异常测度空 间的目标。其步骤如下:

首先, 根据已有的宏观异常分类, 及异常 信息表现等的归纳整理, 鉴于宏观异常表现形 式的复杂性和多样性, 选择典型宏观异常, 构 建本体化的地震宏观异常知识库。

其次, 在后台数据库随机产生与描述宏观 异常群的参量-一种类、总量、空间密集度、 异常幅度相对应的数据, 通过智联网平台以游 戏场景的形式呈现出来。这样, 生成若干组宏 观异常群的场景, 如 10 个。每个场景对应一 个种类 $V$ 的记录, 组成种类集合, 其它参量同 理:

$$
\begin{array}{r}
V=\left\{v_{1}, v_{2}, \ldots, v_{10}\right\}, T=\left\{t_{1}, t_{2}, \ldots, t_{10}\right\}, \\
D=\left\{d_{1}, d_{2}, \ldots, d_{10}\right\}, \quad I=\left\{i_{1}, i_{2}, \ldots, i_{10}\right\} 。
\end{array}
$$

再者, 专家作为一个智能体和计算机进行 交互, 根据模拟游戏里的宏观异常群情景, 对 出现的全部宏观异常的强度做一个整体评判 $G$ 。例如非常显著为 10 , 非常不显著为 0 , 可 在 0-10 之间进行输入。按照人类的认知规律,
我们对某个事物的认知和判断是模糊的, 可以 通过画曲线图的方式进行模糊表达。如果某个 专家认为一个宏观异常群的场景整体强度比 较小, 按照 1-10 的范围大致判断为 $x=3$, 这个 判断是模糊的, 那么就可以如图 1 那样画曲 线，宏观异常强度整体显著性一般，但也不能 完全忽视。这样每个异常场景都可以得到一组 $(x, y)$ 数据, 尽可能地获取专家智能体的认知判 断, 丢失信息相对较少。得到的数据可以利用 信息扩散技术进行处理分析。

最后, 把后台生成的数据种类 $V$ 、总量 $T$ 、 空间密集度 $D$ 、异常幅度 $I$ 作为输入, 专家评 判数据作为输出, 对二者建立模型:

$$
G=F(V, T, D, I)
$$

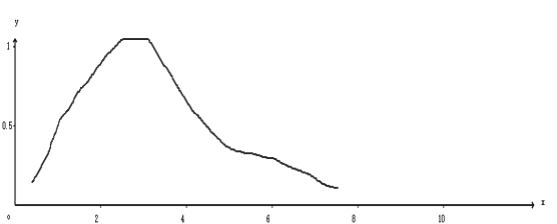

图 1. 模糊输入

这样通过在线智联网平台虚拟宏观异常场景, 专家参与评判, 就可以建立起共识性地震宏观 异常测度空间。

本文选用 Unity3D 开发平台和 C\#开发语 言进行游戏场景的实现, 利用 PHP 语言和 web 服务器端进行交互, 可以在 IOS、Android 和 Windows 上运行。

\section{6. .结论和讨论}

充分发挥互联网的作用, 集众人之所长, 生超 级之智能, 解风险之万千, 把智联网用到社会 各行各业, 进行防灾减灾是我们奋斗的目标。

具有中国特色的群测群防工作, 曾在我国 防震减灾史上发挥过重大作用, 群测得到的宏 观异常数据, 是专业台站数据的有效补充。由 于孕震环境的空间大范围性, 以及宏观异常数 据本身带有的主观性、不确定性等原因，规模 性的宏观异常才有指示意义。目前的宏观异常 研究大多是基于单个或几个震例对出现的宏 观异常数据进行统计分析, 到目前为止, 我们 积累的数据资料还比较少, 只能得到概略、简 单的统计规律。而目前地震的相关研究大多还 


\section{Risk Analysis and Crisis Response in Big Data Era (RAC-16)}

处于初级阶段, 特别是宏观异常在实际工作

中, 更多的是依靠专家经验。

基于此, 本文提出宏观异常群的概念, 定 义合适的参量指标来综合度量宏观异常, 根据 指标模拟宏观异常情景, 建立宏观异常情景评 判智联网, 专家作为高级智能体参与其中, 通 过人机交互对看到的宏观异常情景进行模糊 评判。智联网在后台对得到的结果进行综合处 理, 得到度量宏观异常群的指标种类 $V$ 、总量 $T$ 、空间密集度 $D$ 、异常幅度 $I$ 和专家评判结 果之间的关系，从而建立系统化的测度空间。 下一步就是完善智联网平台, 邀请专家参与进 来, 进行实际案例的实验和分析。

\section{Acknowledgements}

This study was supported by National Natural Science Foundation of China (No.41471424), the State Key Development Program for Basic Research of China "Global change and the environment risk evolution process and comprehensive evaluation model" (No. 2012CB955 402).

\section{致谢}

本研究得到了本研究得到了国家自然科学基 金项目(41171402)和国家重大科学研究计划 “全球变化与环境风险演变过程与综合评估模 型” (编号：2012CB955 402) 的资助。

\section{参考文献}

[1] 刘成龙, 车用太, 王广才.大规模宏观异 常的双重性及其在地震预报中的意义. 地 震地质，2004，26(2):340-346.

[2] 车用太, 鱼金子, 刘成龙. 地震宏观异常 与临震预测成功的希望. 国际地震动态, 2012(6): 138-138.

[3] C.F. Huang, Internet of intelligences in risk analysis for online services, Journal of Risk Analysis and Crisis Response, 2011, 1(2): 110-117.

[4] 艾福利. 自然灾害风险分析智联网服务 平台构建与应用研究. 北京, 北京师范大
学, 2013.

[5] 黄崇福. 用智联网缓解风险分析中的 4 个 问题. 自然灾害学报, 2014, 32(2): 1-7.

[6] C.F. Huang. Internet of Intelligences Can $\mathrm{Be}$ a Platform for Risk Analysis and Management. Human and Ecological Risk Assessment, 2015, 21(5): 1395-1409.

[7] ] F.L. Zeng, C.H. Huang, F.L. Ai. Operation mechanism and interfered information's screening strategy of internet of intelligence. Intelligent Systems and Decision Making for Risk Analysis and Crisis Response, Eds. C.F. Huang, C. Kahraman. Boca Raton, USA: CRC Press, pp. 313-318, 2013

[8] Huang C.F. The measurement of effective knowledge in multiple Internet of intelligences and application in risk assessment. Intelligent Systems and Decision Making for Risk Analysis and Crisis Response, Eds. C.F. Huang, C. Kahraman. Boca Raton, USA: CRC Press, pp. 1-8, 2013.

[9] 艾福利，黄崇福，王蔚丹. 高考志愿填报 风险评估智联网服务平台. 风险分析和 危机反应的创新理论和方法. 黄崇福, 翟国方编。巴黎:Atlantis 出版社, pp.44-49, 2012.

[10] 王蔚丹，黄崇福，艾福利. 基于智联网的 地震预报策略初探. 风险分析和危机反 应的创新理论和方法. 黄崇福, 翟国方 编. 巴黎:Atlantis 出版社, pp. 50-55, 2012 .

[11] 苏妩, 黄崇福, 曾凡雷. 基于智联网的海 洋环境风险评估互联网平台初探。海洋 系统风险分析和管理的新视角,黄崇福, 王春乙, 张㓞编, 巴黎: Atlantis 出版社, pp.14-20, 2014.

[12] 黄崇福. 从概率统计进化到智联网的动 态风险评估. . 风险分析和危机反应中的 信息技术, 黄崇福, 包玉海, 赵思健编. 巴 黎: Atlantis 出版社, pp. 1-8, 2014.

[13] W.D. Wang, S. Qiao, F.L. Zeng, J. Guo. An approach to ranking integrated models for 
risk assessment by using the internet of intelligences. Journal of Risk Analysis and Crisis Response, 2016, 6(1): 2-9.

[14] 中国地震局监测预报司.地震宏观异常摘 编.北京: 地震出版社, 2010.

[15] 祝晔,李荣安.海城 7.3 级地震宏观前兆时 空演化特征与异常机制.地震地质, 1983

[16] 刘五洲, 车用太.张北一尚义地震宏观异 常的震后调查及分析. 地震地质, 1998, 20(2): 183-188.

[17] 高小其, 寇大兵, 宋和平, 等.2008 年 3 月 21 日于田 7.4 级地震宏观异常的调查 与分析.内陆地震, 2009(3):321-328.

[18] 许敦煌. 汶川大地震前宏观异常的现场 调查.地震, 2010, 30 (2): 121-133.

[19] 张小涛, 张永仙, 许敦煌.汶川 8.0 级地震 前后宏观异常现象分析. 地震, 2009, 29(2): 104-117.

[20] 张永久, 吴小平,官致君.汶川地震与松潘 一平武地震的宏观异常对比分析.中国地 震学会成立三十年学术研讨会论文摘要 集,2009.

[21] 付虹, 万登堡, 张立. 云南地区地震宏观 异常特征研究. 地震研究, 2003, 26(3): 209-216.

[22] 卢双苓, 曲保安, 蔡寅, 等. 宏观异常与 地震关系的统计分析. 中国地震, 2015, 31(1): 141-151.

[23] 中国地震局监测预报司. 地震宏观异常 预报方法. 北京: 地震出版社, 1997.

[24] 刘成龙, 车用太, 王广才.大规模宏观异 常的双重性及其在地震预报中的意义. 地 震地质, 2004，26(2):340-346. 